\title{
Design of Education Management System Based on Wireless Self-organizing Network
}

\author{
LIU Xiao-Ling ${ }^{1}$,LI Jun ${ }^{2}$ \\ \{LiuXiaoLing3812@163.com¹,LIjun3812@163.com²\} \\ (1. Institute of Education and Physical Education,Jiangxi Institute of Applied Science and Technology, \\ Nanchang 330100,China; \\ 2.Institute of Education and Physical Education,Jiangxi Institute of Applied Science and Technology, \\ Nanchang 330100,China)
}

\begin{abstract}
In view of the current imperfect construction of education management system in most colleges and universities, the design of education management system based on wireless self-organizing network architecture is proposed.On the basis of the original hardware structure,human-computer interaction design is added,S3C2440 processing chip and EPCS16SI16N storage chip are selected to ensure the normal operation of the system;data transmission bandwidth is reasonably allocated,and the wireless self-organizing network is used to optimize the login interface to realize the design of education management system based on the wireless self-organizing network architecture. The test results show that the original system can not meet the needs of users for knowledge competition and test management,and the designed system improves this defect,and its performance is obviously better than the original system.
\end{abstract}

Keywords: Wireless Self-organizing Network;Dynamic Routing;Educational Management;

\section{Introduction}

The traditional wireless cellular communication network needs the support of fixed network equipment,such as base station,for data forwarding and user service control ${ }^{[1]}$. However,wireless ad hoc network does not need the support of fixed equipment.Each node,i.e.the user terminal,organizes its own network. When communicating,other user nodes forward the data.This kind of network breaks through the geographical limitation of the traditional wireless cellular network,and can be deployed more quickly,conveniently and efficiently.It is suitable for some urgent communication needs, such as the battlefield individual communication system. In reference [2], aiming at the problem of resource optimization in self-organized heterogeneous networks, considering the energy efficiency and the stability of network connections, a scheme of educational resource allocation is proposed. The channel is included into the available channel spectrum pool, and the stability of network connection is guaranteed by the education user selection mechanism based on Morse potential 
energy. In order to improve the energy efficiency of the network, the power is allocated to the education users. In the selection process, the users are ranked according to the Morse potential energy value, and the users with lower potential energy value are preferred to provide services. The results show that the proposed scheme can save the data transmission power loss of education platform, ensure the stability of network connection, and optimize the energy efficiency. Reference [3] designed and built a wireless self-organizing network education management test platform system. By developing the underlying network card driver and routing algorithm protocol module, ordinary PC becomes an independent self-organizing network terminal. At the same time, through monitoring, statistics of data transmission between multiple educational terminals to obtain the measured performance indicators of the network. At present, the platform has implemented the test support for a variety of routing algorithm protocols.

However,wireless ad hoc network also has the disadvantages of limited network bandwidth,poor support for real-time services,and low security.Wireless ad hoc network is a multi hop mobile peer-to-peer network composed of dozens to hundreds of nodes,which adopts wireless communication and dynamic networking.Its purpose is to transmit multimedia information flow with QoS requirements through dynamic routing and mobile management technology.Usually nodes have a continuous energy supply.It is applied to the design of education management system to optimize the system performance.

\section{Hardware design of education management system based on wireless self-organizing network architecture}

\subsection{Design of human-computer interface}

Through the human-computer interface,the communication between users and the system can be realized,and different operations can be performed. In the education management,the form of touch screen is used to select the input of the selected information according to the coordinate position of touch screen or press touch screen. Using touch human-computer interaction function in education management system makes management more convenient and visible.The industrial LCD LQ104VLDG52 is used to realize the display function,which has the advantages of high resolution and high data transmission speed.Its LCD driver module mainly uses programmable CPLD chip to realize the function of data bus and address bus in data exchange between LCD and driver module,so as to ensure high-speed and reliable data transmission ${ }^{[4]}$.In the driver module, the RAM chip is used to realize the data buffer function in the process of data transmission,so as to prevent the data loss of education management data in the process of transmission.

The combination of industrial LCD and driver module can be used as an external device 
to read and write relevant data. The timing diagram of the writing process of the driver module and the LCD is shown in Fig.1.

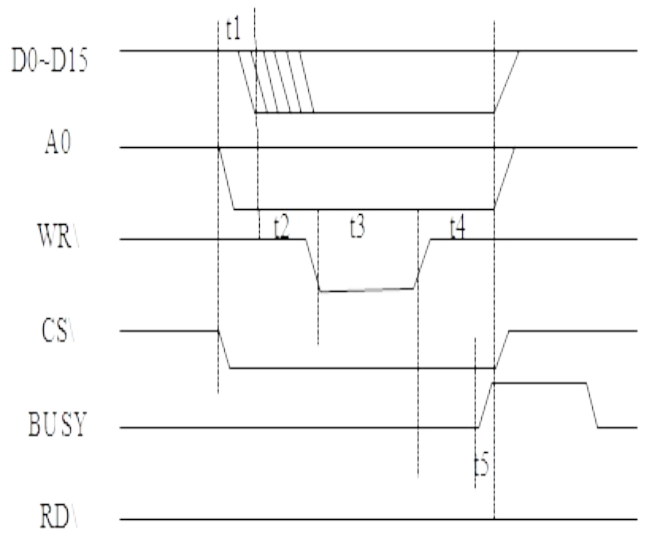

Fig.1.Sequence diagram of the writing process of the LCD screen and the driver module

In Figure 1,t1 represents the minimum effective time from the chip selection signal CS $\backslash$ and address signal A0 of the drive module to the data line D0-D15 of the write process; $t 2$ is the minimum time from data line to write signal $\mathrm{WR} \backslash$ during data writing; $\mathrm{t} 3$ represents the minimum time from $\mathrm{WR} \backslash, \mathrm{RD} \backslash$ line to the end of $\mathrm{CS} \backslash \mathrm{A} 0$; $\mathrm{t} 4$ is the minimum time from the end of WR 1 to the effective time of busy; $t 5$ represents the maximum time of busy ${ }^{[5]}$. The circuit connection structure of each part of human-computer interaction is shown in Figure 2.

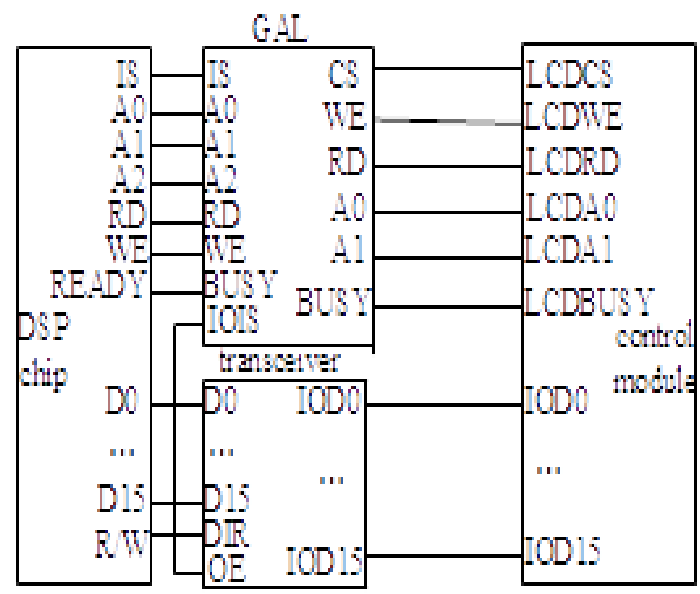

Fig.2. Circuit connection diagram of LCD screen

The three outputs of cal programmable logic make up the address line of LCD and driver module. After logic operation, the input data is connected to the output. The change of output 
signal will not affect the input signal and effectively isolate the signal.

\subsection{Data Processing Chip Selection}

The S3C2440 processor produced by SamSung company based on ARM920T processor core has 5-level pipeline and Harvard structure,and its main frequency reaches 400MHz.It is a 32-bit microcontroller with high cost performance for communication field and system control,which has the following characteristics:(1)the standard working frequency is $400 \mathrm{MHz}$, and the operation rate reaches $450 \mathrm{mps}$.(2)Built in memory management unit and instruction data cache space.(3)On chip oscillator with two PLLs.(4)The chip contains two 16 bit timing/counters with three channels.(5)117 external common I/O and 24 external interrupt sources $^{[6]}$.(6)External bus interface EBI supports SDRAM,nor flash and NAND flash. (7)Integrated LCD controller,support $64 \mathrm{~K}$ color TFT,and a DMA channel dedicated to memory refresh.(8)Four universal synchronous different/step serial transceivers USART,serial peripheral interface SPI.(9)Sdhost supports 1.1 multimedia protocol.(10)Support 10/100M Ethernet interface.According to their own needs,users can choose the hardware devices suitable for the characteristics of the core plate and the bottom plate for welding.The physical figure of core board is shown in Figure 3.

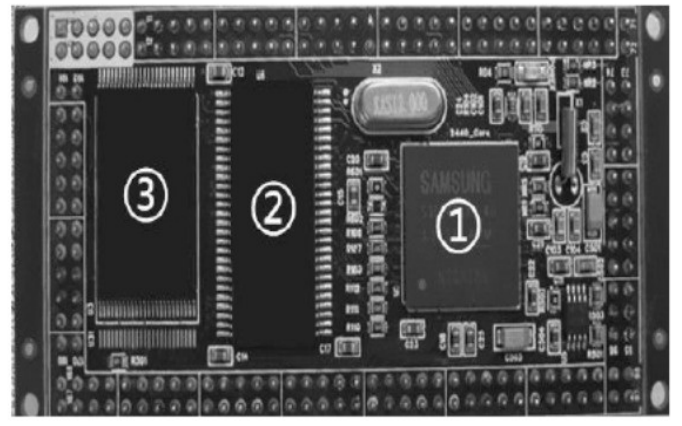

Fig. 3. S3C 2440 Core Plate

As shown in Figure 3, (1) is a Samsung S3C2440 microprocessor chip with the main frequency reaching $400 \mathrm{MHz}$, (2) is the SDRAM memory pin interface. Users can customize suitable memory chips according to their needs, or weld them by themselves. (3) is the reserved NAND flash interface pin. The core board is surrounded by address bus, data bus, JTAG interface pins, GPIO pins of each group and touch screen pin interfaces. These interfaces and backplanes can correspond to the matching backplane slots one by one ${ }^{[7]}$.

\subsection{Circuit design}

Due to the power supply for some precision devices, many devices are sensitive to mutation and rising edge and other variables, so the burr ripple should be as small as possible, 
the load mutation should be as stable as possible, the heating should be as small as possible, the volume should be as small as possible, and the protection function should be complete. The devices used in this system are all small power devices, so using power frequency transformer and linear voltage regulator can completely meet the technical requirements of the system. As the basis of hardware system, power supply can only work normally, and subsequent work can be carried out. See Figure 4 for details of power supply design.

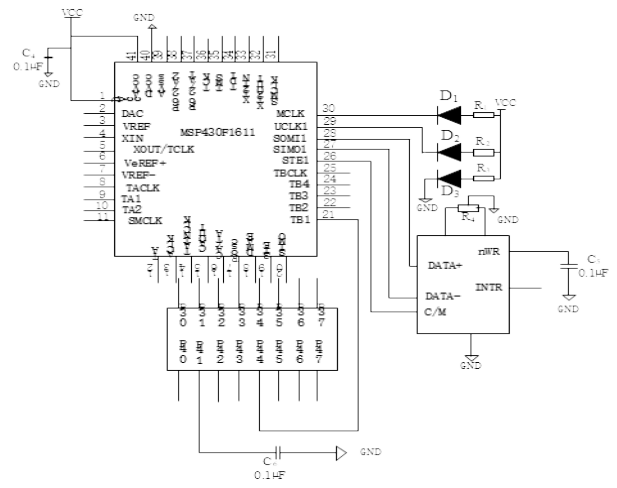

Fig. 4. Power Hardware Circuit Diagram

As shown in Figure 4, 3.3V voltage is used, while $5 \mathrm{~V}$ voltage is generally provided. Here, a power adapter is used to convert $220 \mathrm{~V}$ alternating current into $12 \mathrm{~V}$ DC voltage. $12 \mathrm{~V}$ voltage is converted into $5 \mathrm{~V}$ voltage through $\operatorname{lm} 2596 \mathrm{~s}-5 \mathrm{v}$ chip, and then $5 \mathrm{~V}$ voltage is connected to $1 \mathrm{~m} 10851-3.3 \mathrm{v}$ chip to reduce voltage to $3.3 \mathrm{~V}$.

JTAG is a customized PCB and IC test protocol initiated by several international wellknown electronic manufacturers. It is an international standard test protocol, mainly used for chip internal test, and also supports online programming of advanced logic devices such as FPGA and DSP [6]. Al standard protocol has four main signals: TMS for mode selection, TCK for clock, TDI for data input, and TDO for data output as shown in Figure 5 .

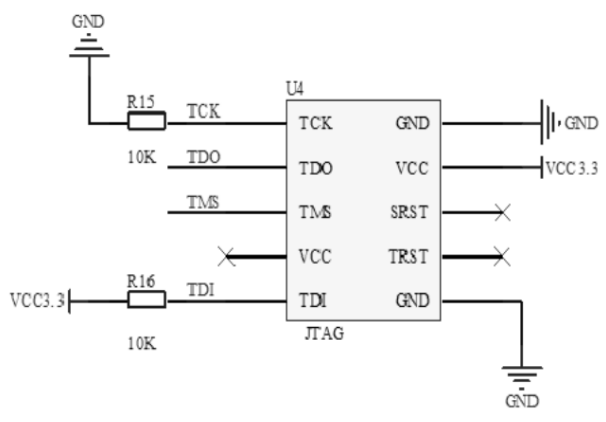

Fig. 5. JTAG hardware circuit diagram 
FPGA based on static memory will lose data information after power failure, so the system needs to reload the configuration file after each power up. In an independent system, the configuration file is usually included in the external flash memory. After power on, FPGA configures the configuration file by loading it into the internal memory. Based on the above reasons, it is necessary to select a memory to store the power on program. The system selects epcs16si16n as the power on program memory chip, as shown in Figure 6.

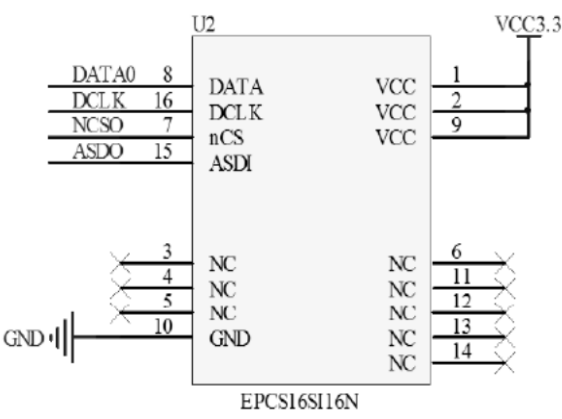

Fig. 6.Circuit diagram of power-up program memory chip

Based on the above, the software part of education management system based on wireless self-organizing network architecture is designed.

\section{Software design of education management system based on wireless self- organizing network architecture}

\subsection{Distribution of data transmission bandwidth}

In the application process of education management system, there are many people online at the same time. In order to ensure the normal operation of the system, determine the priority of the transmission rate, and allocate the network bandwidth resources to the appropriate users.

Set $W_{n s}$ is used to represent the ${ }_{n}$ local user group in network $f$. The users of the local user group are registered users. For the terminals used by users to access the system, the transmission priority is determined by the decision factors of different education management services ${ }^{[8]}$. The priority decision factors are compared to form a matrix of $x \times x$, namely:

$$
X=\left[\begin{array}{cccc}
a_{11} & a_{12} & \cdots & a_{1 x} \\
a_{21} & a_{22} & \cdots & a_{2 x} \\
\cdots & \cdots & \cdots & \cdots \\
a_{x 1} & a_{x 2} & \cdots & a_{x x}
\end{array}\right] \text { (1) }
$$

Where $a$ represents different decision factors. The weighting vector of each decision 
factor is $\partial=\left[\partial_{1}, \partial_{2}, \cdots, \partial_{x}\right]$, which is the eigenvector corresponding to the maximum eigenvalue of matrix $X$. According to this vector, the weighting value of the decision factor can be obtained. In the education management system, the decision factors include throughput, transmission rate, security and cost. Each decision factor compares with each other according to the importance of the influence on the management service, obtains the weighted value of the decision factor, judges the priority of the transmission rate according to the weighted value, and forms the priority decision rules. Based on this, the network bandwidth resources are allocated to different user terminals.

Then the optimal bandwidth allocation matrix is.

$$
G=\frac{W_{n s}(z, c)}{i(z, c)}
$$

In the formula, $z$ represents the user terminal, $v$ represents the network transmission rate, $i(z, c)$ represents the spectral efficiency of the ${ }_{n}$ bandwidth resource in the network $f$ to the user terminal $z$ of the distance education management service. Due to the limited bandwidth resources and the limitation of system capacity, the optimal broadband allocation matrix is constrained by certain conditions, then the constraint conditions of the bandwidth allocation matrix are.

$$
G_{\min } \leq \sum_{f=1}^{F} \sum_{n=1}^{N} G \leq G_{\max }
$$

Where $G_{\min }$ and $G_{\max }$ represent the actual maximum and minimum bandwidth resources of the user terminal supporting the education management service, and meet the needs of the actual education management service through the above constraints.

\subsection{Design of Login Interface Based on Wireless Self-organizing Network Architecture}

The functions of wireless ad hoc network management include performance management, fault diagnosis, service discovery and topology discovery. If we adopt the network management model based on policy and agent, the above network management functions are driven by policy. Performance collection can be active or passive, such as event response, on-demand request, etc ${ }^{[9]}$. The performance analyzer is responsible for processing the basic performance data obtained by the performance collector, and the performance monitoring agent monitors the network nodes through the performance data base and the 
performance event scheduler ${ }^{[10]}$. The fault agent scheduler is responsible for generating, releasing, receiving and destroying the fault detection agent and fault diagnosis agent. Service discovery mainly includes service registration and service query. Topology discovery mainly includes topology report, topology collection, topology timing, etc. it is applied to the application design of login interface, as follows.

The education management subsystem will need to include all links and processes of teaching activities, and its management scope also includes management organization, administrative part, teaching management personnel and teachers, students, other teaching resources and courseware library, etc ${ }^{[11]}$. A good education management subsystem can create a good learning environment and a personalized and intelligent learning atmosphere for students, so a good education management subsystem should include the following functions. (1) Student registration: provide student registration status to facilitate the management of students in the later stage, and ensure the access rights of students, and the access rights of students to teaching resources and courseware library. (2) Student status management: this function is mainly for the management of personal information, grades, course selection, examination, graduation and other aspects of students to ensure the integrity and timeliness of student status. (3) Course management: it mainly includes the establishment and adjustment of major setting, discipline and training plan, and at the same time, it is necessary to ensure the access of visitors within the allowed range, including the course selection of students, etc ${ }^{[12]}$. (4) Management of teaching managers and teachers: to ensure the management of information about teaching managers and teachers, qualification of teaching managers and teachers, assessment and teaching of teaching managers and teachers. (5) Payment function: including the complete realization of the functions of examination, inquiry and statistics of students' tuition fees and examination fees. (6) Administrative document management function: it is convenient for teaching units to release documents related to teaching, including curriculum adjustment, course adjustment, examination information and other functions, and it is also necessary to ensure that these information can be modified and inquired. (7) System setting and maintenance functions: mainly realize the maintenance of the security and stability of the whole system, as well as the backup of relevant data and important data information, the integrity and consistency of data and important data.

User authorization and authentication is an important part of the system, which plays a key role in the security of the whole system ${ }^{[13]}$. We set the browsing mode as the mode and any user with authority can $\log$ in to the system. The user needs to enter the user name and password, and the user authorization and authentication module will review and authenticate the user's qualification to check and determine whether the user is a legal user. If the 
authentication is passed, the user group of the user should be found according to the user name to determine the permissions of the user and the operations that can be performed, and then return to the corresponding page to display the access permissions of the user. In order to ensure the realization of the system security requirements, the password and user authority values are transmitted in an encrypted way ${ }^{[14]}$.In order to facilitate flexible authorization, the system uses the way of connecting with the background database to store the corresponding user permissions and corresponding files one by one, so when the user logs in and issues a request for an operation, the system automatically compares the user information with the records saved in the database ${ }^{[15]}$. If the conditions are met, the requested operation can be allowed, otherwise the access request will be denied. In the login window of the client terminal, the validity of some data and important data is verified first, and then the validity of the user's identity is verified. If it passes the verification, the page corresponding to the user's permission will be returned. Otherwise, the error information will be displayed and the login window will be returned. Of course, the login here is divided into manager login and general user login. The manager is the operator who maintains the normal operation of the system, system security and timely update of information, and is one of the key points of the whole system, the source code of the manager login module is as follows.

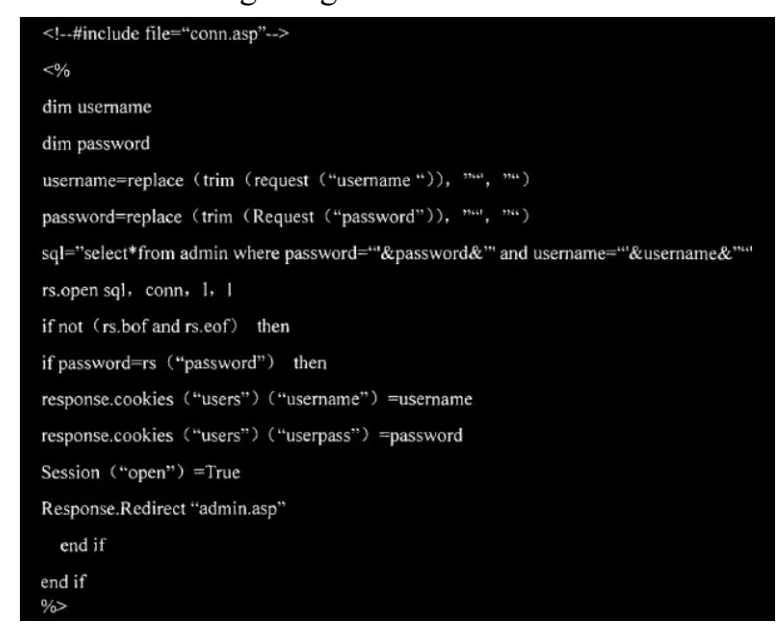

Fig. 7.Administrator login module source code

In the process of user login, firstly, the user name and password entered by the client terminal are submitted to the server. Secondly, the existence of the user is searched in the background database and the matching of the password is compared. The existence of the user and the matching of the password must be satisfied at the same time. So far, the design of education management system based on wireless self-organizing network architecture has 
been completed.

\section{Management system function test}

In order to verify the effectiveness of the education management system based on the wireless self-organizing network architecture, design experiments, test the basic functions of each module of the system, whether it can achieve the design requirements smoothly, and compare the results with the original system, complete the experiment test.

\subsection{Testing process}

In order to meet the needs of the experiment, the hardware environment of the test needs to use Intel HD 4000 or above graphics card, Intel Core i3 $2.4 \mathrm{GHz}$ or above processor, use more than 4GB memory, and the hard disk space is at least $20 \mathrm{G}$ or above. The software environment shall at least meet the requirements of Windows 7/8/10 64-bit or Mac OSX Sierra $(10.12 .6+)$, and the browser version above IE6.0 shall be used. In the above experimental environment, using the Pingdom Tools, using its network and web page detection function, the module function detection of the education management system is completed. The specific operation interface is as follows.

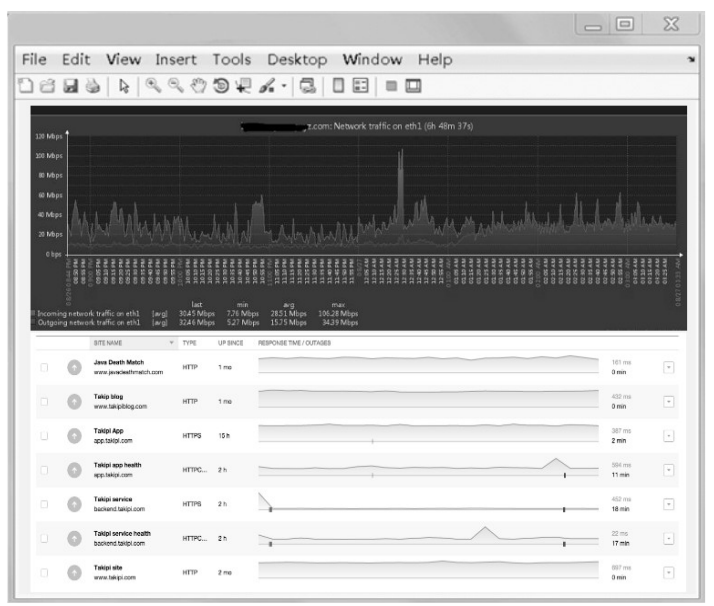

Fig. 8. Operating interface of Pingdom Tools

In the operation process as shown in the figure above, input the relevant information data of a certain college, test whether the relevant functions of user management meet the expectations, and compare with the test results of the original management system to get the results and analyze them.

\subsection{Analysis of test results}

The designed system management test results obtained by Pingdom Tools simulation are compared with the management test results of the original system, as shown in the following figure. 


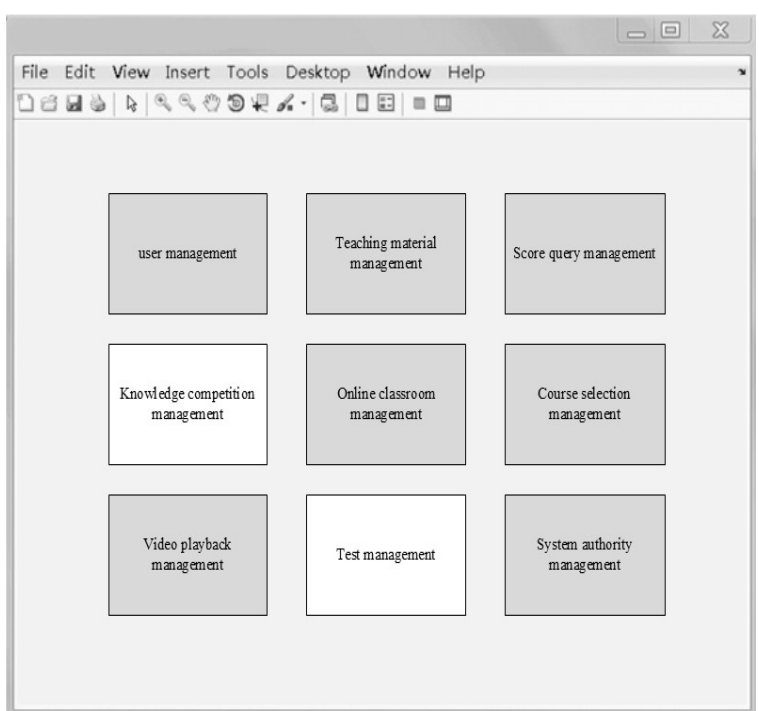

(a)Original system management results

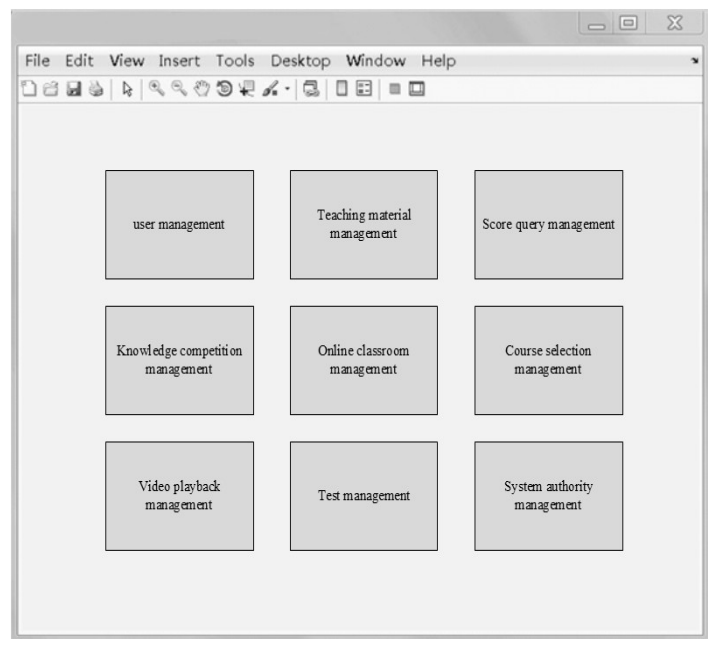

(b)Management results of designed system

Fig. 9. Comparison of test results

In order to ensure the preciseness and reliability of the test, several simulations have been completed during the test. Through the above tests, it can be found that the original system can meet the user's needs in user management, teaching material management, score query management, online classroom management, course selection management, video playback management and system authority management, but in knowledge competition management and test If the test results of these two items are blank, it indicates that the original system has problems in these two items and cannot meet the current user needs. The designed 
management system makes up for this defect and improves the requirements of knowledge competition management and test management. It can be seen that the designed education management system based on wireless self-organizing network architecture can effectively improve some deficiencies of the original system and make the education management work more convenient and efficient.

\section{Conclusions}

In view of the problems of low efficiency and imperfect interaction in the construction of educational administration management system in most universities, this paper proposes a kind of educational administration management system based on wireless self-organizing network structure. Based on the original hardware structure, human-computer interaction design is added. Reasonable allocation of data transmission bandwidth, and use of wireless self-organizing network to optimize the login interface, realize the design of the educational administration management system based on wireless self-organizing network architecture. It has been proved that the application of wireless self-organizing network in the educational administration management system of colleges and universities can optimize the performance of the system and contribute to the educational administration management of colleges and universities.

\section{References}

[1]ZHANG Shaoying.A QoE-based Cross Layer Multi-beam MAC Scheme for Mobile Ad Hoc Networks[J].Telecommunication Engineering,2019,59(07):792-797

[2]LI Mingfei.Multi-channel Access Control Simulation of Self-organizing Network Based on Blockchain[J].Computer Simulation,2019,36(05):480-483

[3]CAI Zhen,LIANG Mangui.An Intra-street Source Routing Mechanism Based on Genetic Algorithm in VANETs[J].Journal of Transportation Systems Engineering and Information Technology,2019,19(04):4349

[4]WANG Fayu,JIANG Yan.Learning interest analysis of users in campus wireless network based on self-organizing neural network and fuzzy C-means clustering algorithm[J].Application Research of Computers,2018,35(01):186-189

[5]WANG Jian.Modeling and Simulation of Large Scale Wireless Sensor Networks Based on OPNET[J].Computer and Digital Engineering,2018,46(11):2305-2309

[6]MA Bin,MAO Buxuan,XIE Xianzhong.Vertical Handoff Algorithm for Reducing Congestion in Ad Hoc Heterogeneous Network[J].Journal of Beijing University of Posts and Telecommunications,2019,42(02):19-24

[7]NIE Song,LIU Ying.Design and Implementation of Enterprise Personnel Management System Based on C\#[J].Software Guide,2018,17(01):126-128 
[8]LIU Yan,XI Hongxia,CAO Jun,et al.A star pattern recognition method based on self-organizing map network and triangle algorithm[J].Chinese Space Science and Technology,2018,38(04):1-10.

[9]QIAO Junfei,AN Ru,HAN Honggui.Design of self-organizing RBF neural network based on relative contribution index[J].CAAI Transactions on Intelligent Systems,2018,13(02):159-167

[10]WEI Qi,LI Shinan,ZHU Yingying.Improved Particle Swarm Optimization Algorithm Optimize the Control Strategy of Family Energy Management System[J].Journal of Harbin University of Science and Technology,2018,23(02):70-77+85

[11]Rajesh M. A Review on Excellence Analysis of Relationship Spur Advance in Wireless Ad Hoc Networks[J]. International Journal of Pure and Applied Mathematics, 2018, 118(9): 407-412.

[12]Hu X, Cheng J, Zhou M, et al. Emotion-aware cognitive system in multi-channel cognitive radio ad hoc networks[J]. IEEE Communications Magazine, 2018, 56(4): 180-187.

[13]Kretchmar K, Sondel B, Ferrare J J. The power of the network: Teach for America' s impact on the deregulation of teacher education[J]. Educational Policy, 2018, 32(3): 423-453.

[14]da Rosa Righi R, Rodrigues V F, Rostirolla G, et al. A lightweight plug-and-play elasticity service for self-organizing resource provisioning on parallel applications[J]. Future Generation Computer Systems, 2018, 78(10): 176-190.

[15]Chatterjee S, Davies M J, Heller S, et al. Diabetes structured self-management education programmes: a narrative review and current innovations[J]. The Lancet Diabetes \& Endocrinology, 2018, 6(2): 130-142. 